\title{
An Improved Encapsulation Method for Cryopreserving Hepatocytes for Functional Transplantation Using a Thermo-reversible Gelation Polymer
}

\author{
KOSUKE YAMADA ${ }^{1}$, TAKESHI AOKI ${ }^{1}$, YUTA ENAMI ${ }^{1}$, YOSHIHIKO TASHIRO ${ }^{1,2,3}$, \\ ZIN ZEHAOU ${ }^{1}$, TOMOTAKE KOIZUMI ${ }^{1}$, TOMOKAZU KUSANO ${ }^{1}$, KAZUHIRO MATSUDA ${ }^{1}$, \\ YUSUKE WADA $^{1}$, HIDEKI SHIBATA ${ }^{1}$, KODAI TOMIOKA ${ }^{1}$, KRIS SIRIRATSIVAWONG ${ }^{4}$, \\ ROBERT M. HOFFMAN ${ }^{2,3}$ and MASAHIKO MURAKAMI ${ }^{1}$ \\ ${ }^{1}$ Department of Gastroenterological and General Surgery, School of Medicine, Showa University, Tokyo, Japan; \\ ${ }^{2}$ Department of Surgery, University of California, San Diego, CA, U.S.A.; \\ ${ }^{3}$ AntiCancer Inc, San Diego, CA, U.S.A.; \\ ${ }^{4}$ Department of Medical Education, School of Medicine, Showa University, Tokyo, Japan
}

\begin{abstract}
Background/Aim: Thermo-reversible gelation polymer (TGP) can be converted into a gel state upon warming and liquid upon cooling. The present study aimed to demonstrate a new method for cryopreservation and encapsulation of rat hepatocytes using a TGP and their successful transplantation. Materials and Methods: The isolated rat hepatocytes were microencapsulated using TGP, and stored in liquid nitrogen. After cryopreservation, hepatocytes were cultured. Moreover, hepatocytes were transplanted into the spleen without a TGP capsule. Results: The viability of hepatocytes that were cryopreserved in TGP was $71.2 \pm 2.3 \%$. The hepatocytes demonstrated adequate survival, maintained their hepatic function in culture, and expressed albumin after transplantation to the rat spleen. Conclusion: We demonstrated a cryopreservation method of rat hepatocyte encapsulation using a TGP gel in the hydrogel state which subsequently allowed successful transplantation of unencapsulated hepatocytes in a sol state TGP gel at low temperature.
\end{abstract}

The number of liver transplantation in patients with endstage liver disease has been increasing. Unfortunately, there

This article is freely accessible online.

Correspondence to: Takeshi Aoki, MD, Ph.D., Department of Gastroenterological and General Surgery, School of Medicine, Showa University, 1-5-8, Hatanodai, Shinagawa-ku, Tokyo, 1428666, Japan. Tel: +81 337848541, Fax: +81 337845835, e-mail: takejp@wb4.so-net.ne.jp

Key Words: Hepatocyte transplantation, microencapsulation, cryopreservation, thermo-reversible gelation polymer (TGP), function, rats. is a shortage of organ donors for these patients, which prevents treatment via transplantation.

A potential alternative to liver transplantation for patients with severe liver disease is hepatocyte transplantation (HT) (1-7). However, the transplantation of allogeneic hepatocytes is also limited due to a shortage of liver donors and poor proliferative potential of hepatocytes in vitro. Therefore, a method whereby large quantities of hepatocytes can be preserved for a long period, thereby providing a convenient and easily accessible supply, is highly desirable.

Cryopreservation of hepatocytes is a standard method for long-term preservation $(8,9)$. However, standard cryopreservation procedures can severely damage hepatocytes. Therefore, new strategies for long term storage with adequate protection of hepatocytes are needed $(8,9)$. Although utilizing a programmable freezer with a special medium is effective for cryopreservation of hepatocytes, new methods are still needed to maximize cell viability and maintain normal hepatic function. Our group has previously demonstrated an encapsulation technique as a new method for long-term cryopreservation of rat and human hepatocytes (10).

Although the encapsulation technique was effective for cryopreserving hepatocytes, post-transplantation was problematic because the capsule can easily rupture in the spleen, resulting in leakage of the donor cells. Moreover, the capsular membrane inhibits cell-to-cell physical contact, which may impact normal cellular function.

To address this issue, we used a thermo-reversible gelation polymer (TGP) (Mebiolo Gel ${ }^{\circledR}$ Mebiol Inc., Hiratsuka, Japan). TGP is a unique polymer that remains in solution (sol) state at low temperatures $\left(0^{\circ} \mathrm{C}-15^{\circ} \mathrm{C}\right)$, but can change into a gel state at temperatures higher than $25^{\circ} \mathrm{C}$. This polymer enables encapsulation during cryopreservation to 

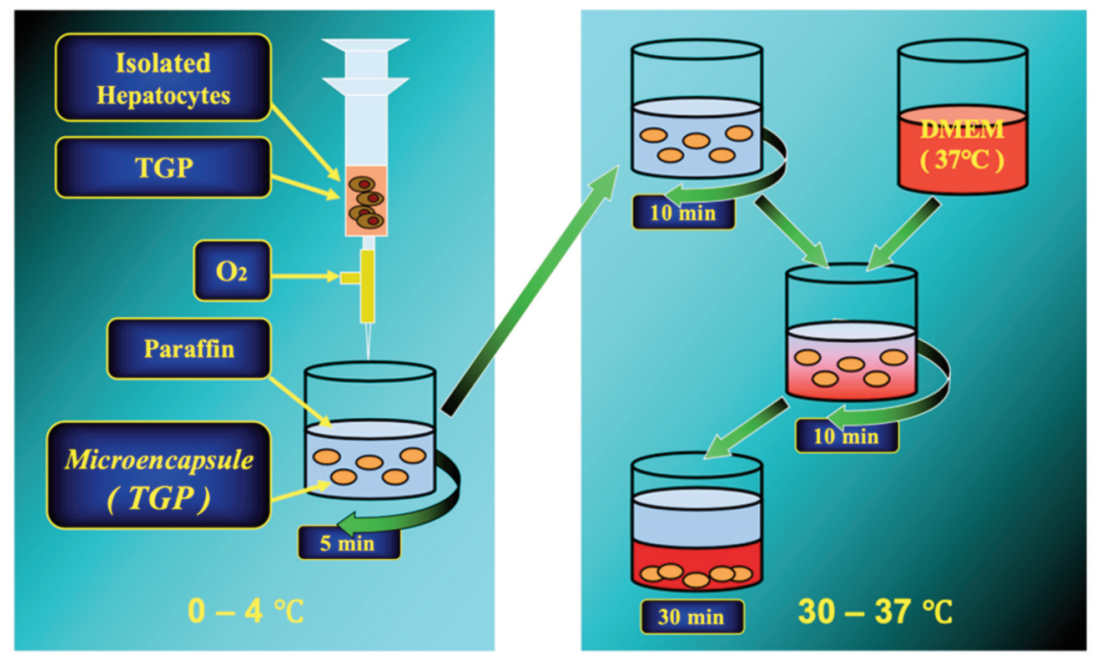

Figure 1. Microencapsulation technique.

protect the hepatocytes from cryoinjury, and easy removal of the capsule after transplantation of the cryopreserved cells.

The present study provides a cryopreservation method of rat hepatocytes encapsulated using TGP in the gel state, and viable recovery of unencapsulated hepatocytes in the sol state at a low temperature with subsequent functional transplantation.

\section{Materials and Methods}

Animals. Sprague-Dawley (SD) rats (250-350 g, Saitama Experimental Animals Supply, Saitama, Japan) were housed under 12-hour light/dark cycles for over 7 days before surgery. Animal studies were performed at the Laboratory Animal Center of Showa University School of Medicine and approved by the Showa University Ethics Committee for Animal Care and Use. All aseptic operations were under general anesthesia.

Rat hepatocytes. Rat liver cells were harvested by a two-step EDTA/collagenase digestion in situ as previously described (11). Following enrichment through a Percoll density gradient, cell viability was determined to be greater than $95 \%$, assessed using the trypan-blue dye-exclusion test.

Preparation of thermo-reversible gelation polymer $(T G P)$. Under a clean-air laminar-flow hood, a flask containing $10 \mathrm{ml}$ of sterile Mebiol gel (supplied by Mebiol Gel Inc., Ltd, Hiratsuka, Japan from Nichi In Drugs \& Devices (Pvt) Ltd, Chennai, India) was opened and $10 \mathrm{ml}$ Eagle's Minimum Essential Medium (MEM) containing gentamicin $50 \mathrm{mg} / \mathrm{ml}$ ciprofloxacin $10 \mathrm{mg} / \mathrm{ml}$ and $10 \%$ fetal bovine serum (FBS) (all obtained from HiMedia, Mumbai, India) were included. The gel was dissolved in the medium at 4 to $8^{\circ} \mathrm{C}$ within $72 \mathrm{~h}$.

Hepatocyte isolation and microencapsulation. Figure 1 illustrates the microencapsulation technique. Hepatocytes $\left(1 \times 10^{7}\right)$ suspended in a small amount of Dulbecco's MEM (DMEM) were mixed with $5 \mathrm{ml}$ of TGP/MEM at $4^{\circ} \mathrm{C}$. Droplets containing hepatocytes in TGP/MEM formed microcapsules by extrusion through a $21 \mathrm{G}$ needle, followed by placing them in a beaker containing $10 \mathrm{ml}$ paraffin oil (PO) (No. 7162, Merck, Darmstadt, Germany) and rotation at $4^{\circ} \mathrm{C}$. This resulted in the solidification of the microcapsules, which became further water-insoluble after being heated above the soluble-gel transition temperature (SGTT) for $10 \mathrm{~min}$. The beaker containing $10 \mathrm{ml}$ DMEM was immersed in a $37^{\circ} \mathrm{C}$ water bath and shaken gently to precipitate the microcapsules. The microcapsules were then washed in DMEM, and those with diameters between 150 and $300 \mu \mathrm{m}$ were spontaneously formed.

Cryopreservation of hepatocytes. Isolated encapsulated rat hepatocytes were placed in a cryopreservation medium consisting of $10 \%$ dimethyl sulfoxide (DMSO), 10\% FBS and 80\% Dulbecco's Modified Eagle Medium (DMEM). Aliquots of encapsulated hepatocytes were distributed in $1.8 \mathrm{ml}$ freezing vials (Nunc, Denmark), which were immediately frozen in liquid nitrogen for storage.

In order to thaw the encapsulated hepatocytes, the vials were placed in a $37^{\circ} \mathrm{C}$ water bath until the liquid in the tube was melted. The encapsulated hepatocytes were pipetted out and were washed with DMEM containing 10\% FBS. The aliquots were then transferred to DMEM culture medium in dishes.

Culture of cryopreserved hepatocytes. Cryopreserved hepatocytes $\left(1 \times 10^{7}\right)$ were placed in DMEM enriched with $0.2 \%$ bovine serum albumin, 10\% FBS, $10 \mathrm{mM}$ nicotinamide, $25 \mathrm{mM} \mathrm{NaHCO}, 20$ $\mathrm{ng} / \mathrm{ml} \mathrm{EGF}, 10^{-7} \mathrm{M}$ dexamethasone, $30 \mu \mathrm{g} / \mathrm{ml}$ L-proline, $0.5 \mathrm{mM}$ glutamine, $1 \mathrm{mg} / \mathrm{ml}$ galactose, $20 \mathrm{mM}$ HEPES, $0.1 \mathrm{mM}$ L-ascorbic acid 2-phosphate (Acs-2P), $0.5 \mu \mathrm{g} / \mathrm{ml}$ insulin-transferrin-seleniumX 51500 (ITS 51500, Life Technologies, Rockville, MD, USA), 50 $\mu \mathrm{g} / \mathrm{ml}$ streptomycin and, $100 \mu \mathrm{g} / \mathrm{ml}$ penicillin, and transferred to a suspension-culture dish $(60 \mathrm{~mm} \times 15 \mathrm{~mm}$, Corning Incorporated, Corning, NY, USA) and cultured for 28 days. Cell cultures were incubated at $37^{\circ} \mathrm{C}$ in a humidified atmosphere containing $5 \% \mathrm{CO}_{2}$, and the medium was changed every other day. 
Cell viability assays. The trypan-blue exclusion test was used to examine the viability of hepatocytes before and at specific times after cryopreservation as previously described (12).

Measurement of albumin production in culture. Albumin production and urea synthesis were measured, in the 3 groups below, in order to evaluate hepatic function in freeze-thaw and post-thaw culture conditions. The groups were: Group A: Non-cryopreserved rat hepatocytes; Group B: Non-cryopreserved rat hepatocytes with TGP; Group C: Cryopreserved rat hepatocytes with TGP.

Albumin production was analyzed in the medium on days 1, 3, $5,7,10$, and 14 of culture, by utilizing a rat albumin ELISA kit (Shibayagi Co. Ltd., Gunma, Japan). The albumin levels in each of the samples in the medium was also determined.

Measurement of urea nitrogen synthesis in culture. Ammonium chloride $(2.0 \mathrm{mM})$ was added in the culture medium on days 1,3 , $5,7,10$, and 14 after plating. Six hours following the addition of ammonium chloride, the urea nitrogen concentration was measured using a urea-nitrogen diagnostic kit (Bioparmigen, San Diego, CA, USA). The extinction coefficient was measured with a spectrophotometer for measuring urea synthesis (UV-1200, Shimadzu Co., Ltd, Kyoto, Japan).

Histological assessment. Transplanted microencapsulated hepatocytes were fixed in $10 \%$ formaldehyde. Immunocytochemical staining of intracellular albumin in encapsulated rat hepatocytes was performed on paraffin sections. Immunocytochemical staining of intracellular albumin was demonstrated in cultured hepatocytes fixed in $10 \%$ formaldehyde. Briefly, the slides were incubated with a peroxidase blocking reagent (DAKO, Milan, Italy) for $10 \mathrm{~min}$, washed, and incubated with a primary rabbit polyclonal antibody against rat albumin (Omega Scientific, Inc. Tarzana, CA, USA) for $30 \mathrm{~min}$ at room temperature. Subsequently, a secondary antibody (Omega Scientific, Inc. Tarzana, CA, USA) and a visualization reagent (DAKO) were added to the slide, which was incubated at room temperature for $30 \mathrm{~min}$. Diaminobenzidine (DAB) was used as the chromogen.

$R T$-PCR analysis of albumin $m R N A$. Total RNA was isolated from hepatocytes of five different SD rats by using the acid guanidinium thiocyanate (GTC)-phenol-chloroform extraction method. The liver tissue was homogenized in GTC solution (4.0 M GTC containing 25 $\mathrm{mM}$ sodium citrate, $\mathrm{pH} 7.0,0.5 \%$ sarkosyl, and $0.1 \mathrm{M} 2$ mercaptoethanol in a ratio of $10 \mathrm{ml}$ GTC solution per $100 \mathrm{mg}$ liver tissue). The RNA was extracted twice into phenol and chloroform (1 volume phenol/0.2 volumes chloroform/1 volume GTC solution) and precipitated with isopropanol at room temperature. The precipitated pellet was then dissolved in $0.3 \mathrm{ml}$ of GTC solution and precipitated with isopropanol at $-20^{\circ} \mathrm{C}$, washed with ice-cold $80 \%$ ethanol and dissolved in an appropriate volume of diethylpyrocarbonate-treated water. The RNA yield, purity, and integrity were analyzed by the 260 $\mathrm{nm} / 280 \mathrm{~nm}$ absorbance ratio (>1.6), which was confirmed by electrophoresis on $1.0 \%$ agarose/formamide gels.

One $\mu \mathrm{g}$ sample of total RNA was used for RT-PCR. The nucleotide sequences of the RT-PCR primers and the length of the amplified products were as follows: rat albumin, (CTGATATCTGCAC ACTCCCA and TCAGTGGCGAAGCAGTTATC, 181 bp). RT-PCR was performed under the following conditions according to the manufacturer's instructions (Toyobo, Osaka, Japan): 1 cycle at $60^{\circ} \mathrm{C}$ for $30 \mathrm{~min}, 1 \mathrm{cycle}$ at $94^{\circ} \mathrm{C}$ for $2 \mathrm{~min}, 40$ cycles at $91^{\circ} \mathrm{C}$ for $30 \mathrm{~s}$, annealing at $62^{\circ} \mathrm{C}$ for $30 \mathrm{~s}, 72^{\circ} \mathrm{C}$ for $30 \mathrm{~s}$, which was followed by a final extension at $72^{\circ} \mathrm{C}$ for $10 \mathrm{~min}$. Amplified PCR products obtained from five different rats were loaded onto a $2.0 \%$ agarose gel.

Hepatocyte transplantation (HT) study. Hepatocytes from male SD rats were used as transplant donor cells, and male Nagase analbuminemic (NA) rats were used as recipients. With the rats under general anesthesia (achieved with diethyl ether), a small left subcostal incision was made to expose the spleen in each NA rat. Approximately $2 \times 10^{7}$ cryopreserved unencapsulated hepatocytes in TGP, suspended in 1.5 $\mathrm{ml}$ of phosphate-buffered saline, were injected into the spleen through an 18-gauge needle. Hemostasis at the injection site was achieved with 2-0 silk sutures. Each group was subcutaneously injected with $1 \mathrm{mg} / \mathrm{kg}$ FK506 (Astellas Pharmaceutical Co., Ltd, Tokyo, Japan) daily for one week before and two weeks after HT, then once a week until the end of the experiment. Rats were sacrificed at various times following HT, and the spleen was collected.

Measurements of albumin production in NA Rats. In order to evaluate the efficacy of HT, the levels of albumin were determined in serum. Samples were measured using a standard blood chemistry method, automatic analyzer.

Morphologic studies of transplanted hepatocytes in the spleens of $N A$ rats. The spleens that underwent HT were processed for histology. Paraffin-embedded sections $(4 \mu \mathrm{m})$ were stained with $\mathrm{H} \& \mathrm{E}, \mathrm{PAS}$, and immunocytochemistry for albumin.

Periodic acid-Schiff's (PAS) staining. Transplanted hepatocytes were fixed in $10 \%$ formaldehyde for $30 \mathrm{~min}$, oxidized in $10 \mathrm{~g} / \mathrm{l}$ periodic acid for $10 \mathrm{~min}$ and rinsed three times in distilled water (dH2O). The cells were then placed in Schiff's reagent for $10 \mathrm{~min}$, rinsed in $\mathrm{dH}_{2} \mathrm{O}$ for $10 \mathrm{~min}$, and stained with $\mathrm{H}-\mathrm{E}$ for 2 min. After mixing with $1 \%$ alcohol/HCL, the cells were rinsed in $\mathrm{dH} 2 \mathrm{O}$ and examined using a light microscope.

Statistical analysis. All values are presented as mean \pm standard deviation (SD). The Student's $t$-test was performed. $p<0.05$ was considered significant.

\section{Results}

Viability of cryopreserved rat hepatocytes. The viability of hepatocytes in Group A, B, and C before cryopreservation was $86.7 \pm 4.5 \%, 85.3 \pm 5.1 \%$ and $85.3 \pm 5.1 \%$ respectively, and more than $80 \%$ of the cryopreserved microencapsulated rat hepatocytes were viable 48 hours after cryopreservation. The viability of cryopreserved unencapsulated hepatocytes in the sol state TGP gel at low temperature was $71.2 \pm 2.3 \%$. Comparing the viability between before cryopreservation and several points after cryopreservation, no significant differences were noted.

Measurement of albumin production. Albumin production by the hepatocytes in each group was analyzed throughout the cell culture period ( $n=5$ in each group) (Figure 2). In Groups $\mathrm{B}$ and $\mathrm{C}$, the albumin levels were maintained at a high level, with no statistically-significant differences between them. In 


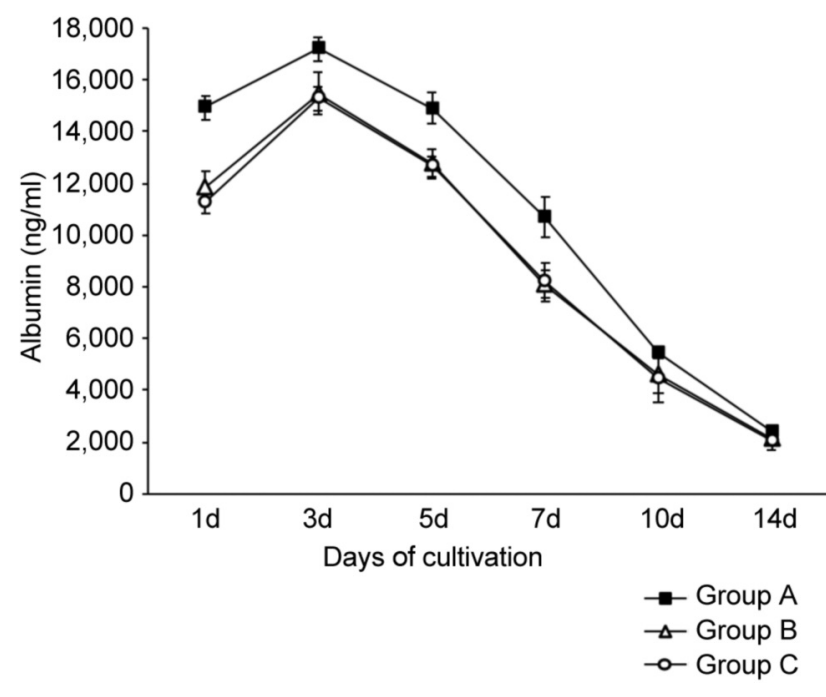

Figure 2. Albumin production by cultured hepatocytes at various time points during cell cultivation ( $n=5$ in each group).

group A, albumin production was lower than in the other groups throughout the experimental period, except for one day. Following five days of culture, the albumin levels in all groups gradually decreased and stabilized at a low plateau. At that time point, no statistical differences between the groups were noted.

Measurement of urea synthesis. Urea synthesis by hepatocytes was evaluated throughout the cell culture period $(n=5)$ (Figure 3). In Groups A and B urea synthesis peaked at 3 days after culture began and then gradually decreased. No statisticallysignificant differences were observed between groups A and B at any time point. Urea synthesis in Group $\mathrm{C}$ reached a maximum level 3 days after culture began and then gradually decreased.

Morphological findings. Before cryopreservation and at various times after cryopreservation, rat hepatocyte morphology was well-preserved. After encapsulation, the capsules retained their round shape, and the hepatocytes inside the capsules had a normal appearance as observed by phase-contrast microscopy, just before cryopreservation (Figure 4). The presence of albumin was demonstrated using a specific cytoplasmic stain. The entrapped hepatocytes stained strongly positive for albumin, and this persisted after 48 hours of cryopreservation.

Culture of cryopreserved rat hepatocytes. The cryopreserved rat hepatocytes were functional after one week of culture. This was confirmed through immunocytochemistry, which showed that hepatocytes were strongly positive for albumin, throughout the study (Figure 5A and B).

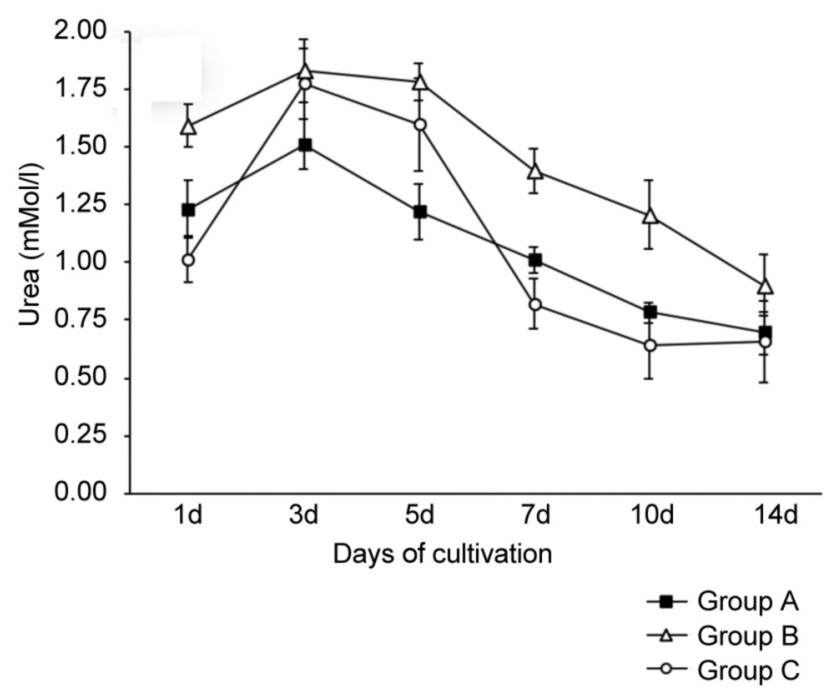

Figure 3. Urea synthesis by cultured hepatocytes at various time points during cell cultivation ( $n=5$ in each group).

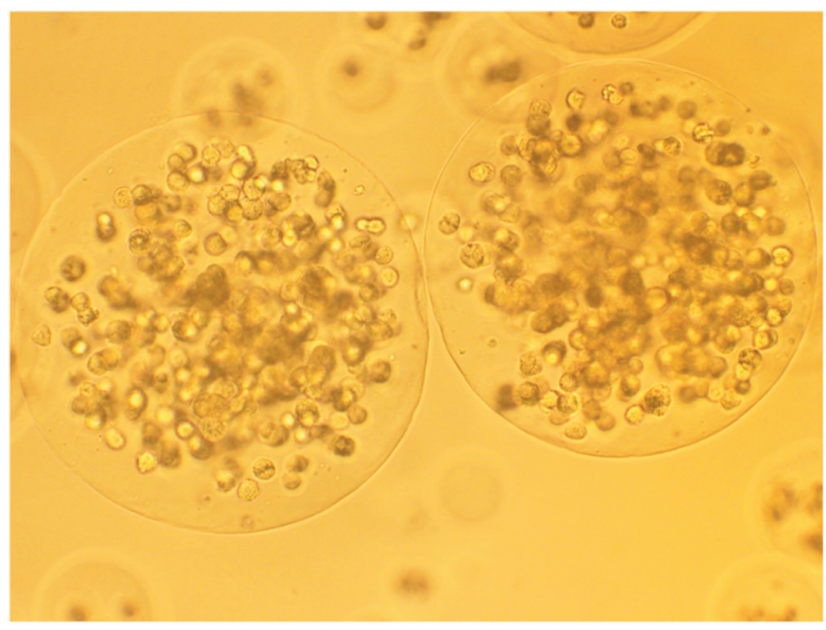

Figure 4. Morphology of isolated rat hepatocytes with TGP before cryopreservation (original magnification $\times 200$ ).

RT-PCR analysis of cultured hepatocytes. The albumin mRNA levels in cultured hepatocytes of Groups A, B, and C after $0,1,3,5,7,10$, and 14 days in culture are shown in Figure 6. The measured liver-specific markers including albumin, were expressed at high levels in Groups A, B, and $\mathrm{C}$, at all points throughout the culture period (Figure 6).

Intrasplenic transplantation of cryopreserved hepatocytes. The serum albumin levels were measured at various time points after HT to the spleen of NA rats (Figure 7). Before HT, the baseline levels of serum albumin in each group were at a minimum, with no statistically-significant differences 

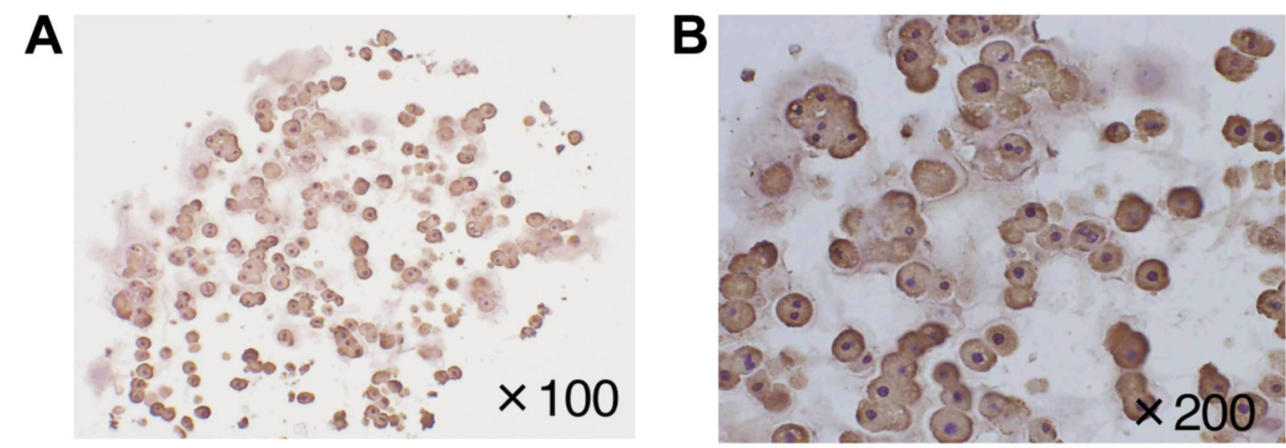

Figure 5. Immunochemical staining for rat albumin in cryopreserved hepatocytes with TGP (Group C) 7 days after cultivation [original magnifications $\times 100(A)$ and $\times 200(B)]$.

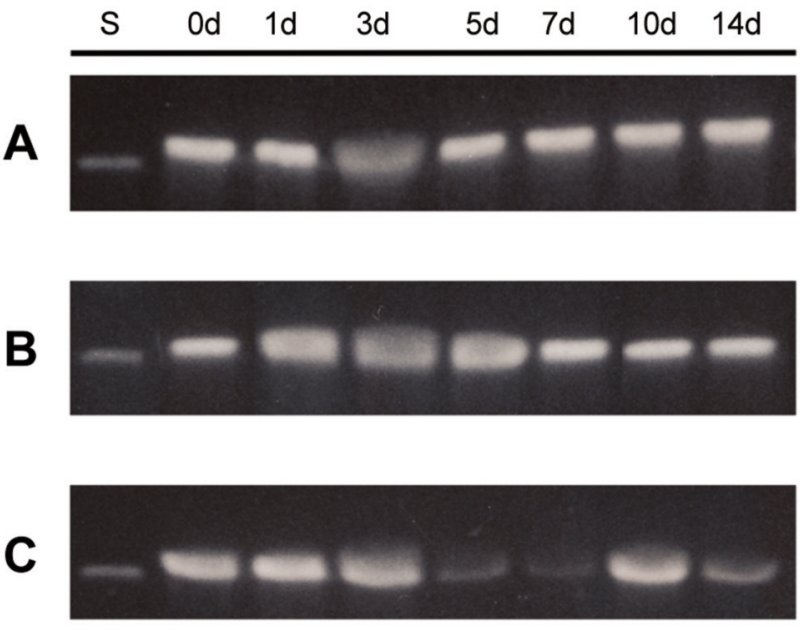

Figure 6. RT-PCR analysis of each group after 0, 1, 3, 5, 7, 10, and 14 days of culture. (A); Group A. (B); Group B. (C); Group C. Lane S: Standard $200 \mathrm{bp}$. Lanes 1-7: Hepatocyte albumin mRNA from 0, 1, 3, $5,7,10$, and 14 days of culture.

between them $(n=5)$. After HT, the serum albumin level increased significantly in all groups, and was maintained at a high plateau for at least 10 days. Although there were statistically-significant differences on day 3 and 10, as well as 2 weeks after HT, no statistically-significant differences were noted between groups at the peak point on day 7 or at 8 weeks after HT (Figure 7).

Morphologic studies of transplanted hepatocytes in the rat spleens of NA rats. The transplanted cryopreserved hepatocytes in the spleen parenchyma of the NA rats were observed to be microscopically viable 56 days after HT in every group (Figure 8A-I). PAS staining analysis showed that the engrafted hepatocytes in Groups A, B, and C were positive for PAS 56 days after HT, demonstrating that the engrafted

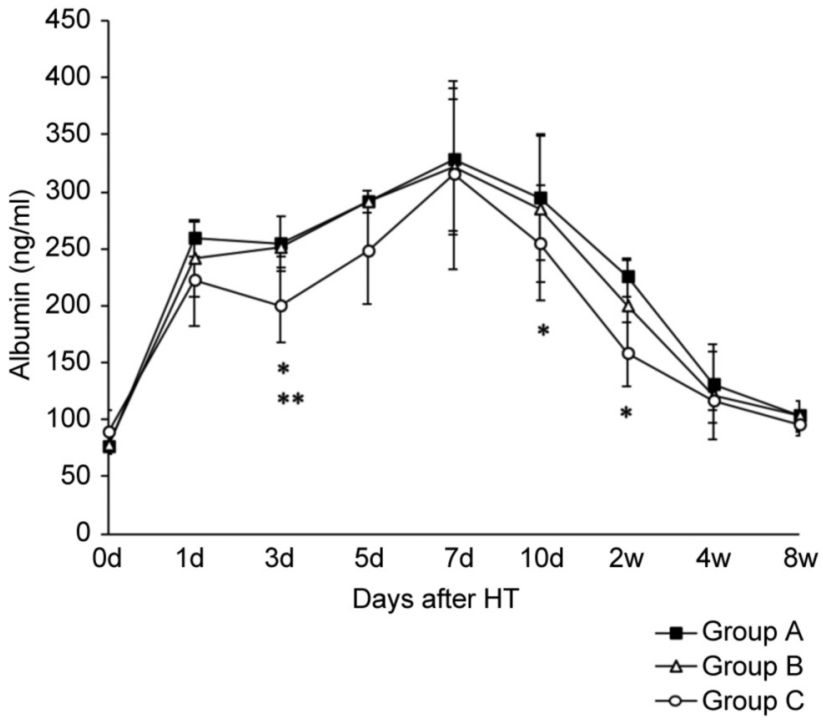

Figure 7. Albumin production at various time points after HT of hepatocytes to NA rats ( $n=5$ in each group). * $p<0.05$ (Group A versus Group C). **p $<0.05$ (Group B versus Group C). HT: Hepatocyte transplantation.

hepatocytes were producing glycogen, an important function specific to hepatocytes. Immunocytochemical analysis of albumin also demonstrated that the engrafted hepatocytes in all groups were positive for albumin, which was seen 56 days after HT.

\section{Discussion}

Koebe et al. developed a method that allows for cryopreservation of porcine hepatocytes by immobilizing them in a collagen gel on a tissue culture surface (13). Dixit (14) and Guyomard (15) described an encapsulation technique that utilizes an alginate-bead gel, which was found 


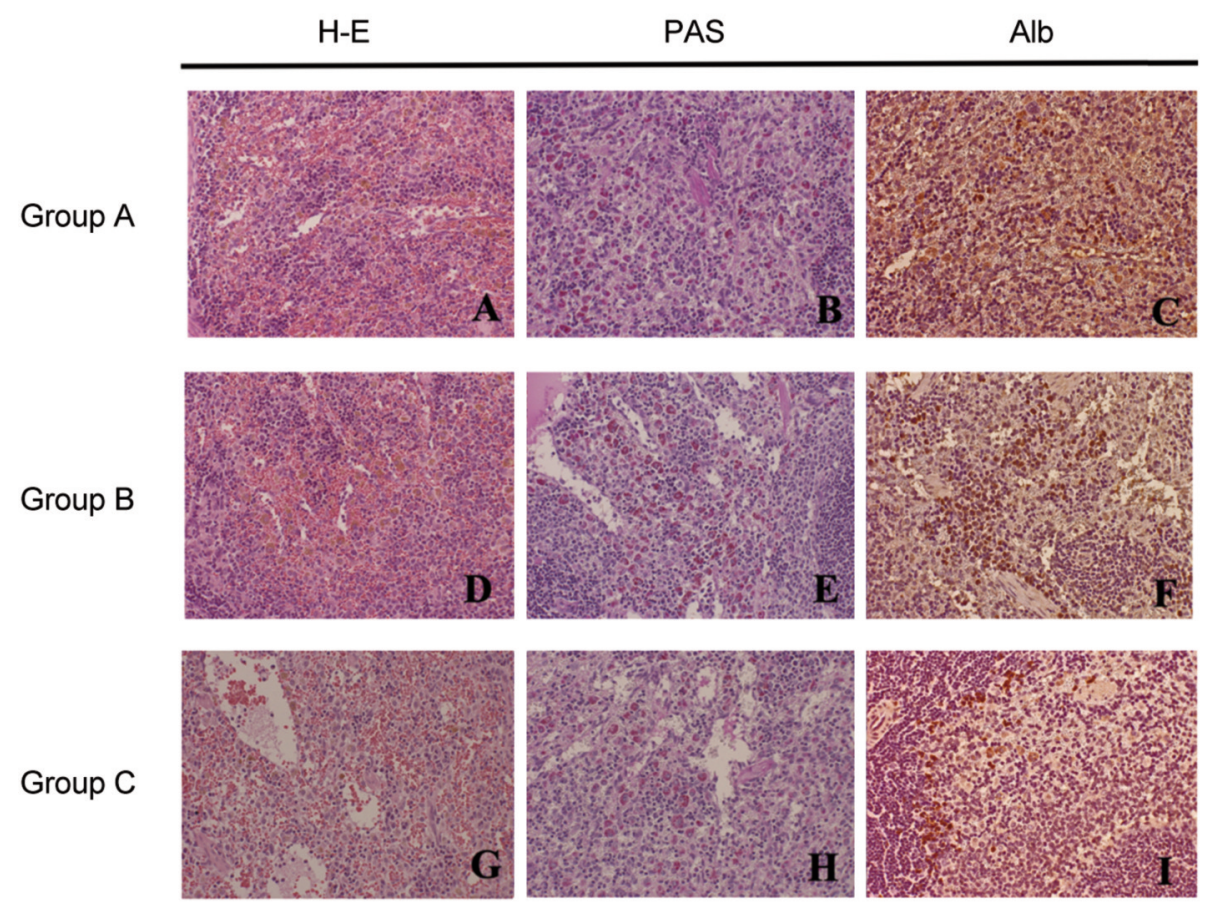

Figure 8. Morphologic studies of transplanted hepatocytes in the spleens of NA rats 56 days after HT. (A-C); Group A. (D-F); Group B. (G-I); Group C. (A, D, and G); Hematoxylin and eosin staining (original magnification $\times 100)$. (B, E, and H); Periodic acid-Schiff's (PAS) staining (original magnification $\times 100)$. (C, F and I); Immunocytochemical staining for albumin (original magnification $\times 100)$. HT: Hepatocyte transplantation.

to be useful and beneficial for cryopreservation of hepatocytes. When these encapsulated hepatocytes were thawed and transplanted into Gunn rats with hyperbilirubinemia, the rats demonstrated improvement in serum bilirubin levels, which was maintained for 30 days following HT (14). Guyomard et al. evaluated the survival function of calcium-alginate-bead-entrapped rat hepatocytes. They analyzed both phase I and phase II enzyme activities before and after cryopreservation and demonstrated that all functions tested were well-preserved (15). We have previously reported successful cryopreservation of human and rat hepatocytes after encapsulation in alginate/poly-L-lysine. Using a cryomicroscope to observe the response of the cells subjected to the freezing-thawing process (10), cell viability, organic-anion transporter expression, and expression of drugmetabolizing enzymes were shown to be well-preserved at different time points after cryopreservation. Morphologically, the entrapped hepatocytes retained their normal appearance and had well-preserved nuclei following 90 days of cryopreservation. Similar success was achieved with cryopreserved encapsulated human hepatocytes, which also retained viability and hepatic function (10). However, the use of encapsulated hepatocytes was generally limited by low mechanical strength, degeneration of capsules over a longterm period, and frequent induction of inflammatory responses. In the present study, we described the development of a new and simple technique for the cryopreservation of hepatocytes after encapsulation using TGP. The capsule itself was removed to obtain excellent cell survival after transplantation. TGP is a chemically-synthesized biocompatible polymer material with unique chemical properties. Below a low critical solution temperature (LCST), it becomes a liquid, and above this temperature, it becomes a solid. Using TGP, we were able to cryopreserve encapsulated hepatocytes without causing hepatocyte dysfunction after capsule removal following thawing. Our results indicated that the viability of hepatocytes was maintained at $85.3 \%$ compared to $86.7 \%$ just after capsulation following their isolation. The decrease in viability was only $14.1 \%$ after cryopreservation. There were no apparent differences in cell morphology between Group B and Group C, and albumin production and urea synthesis were maintained at levels similar to those of non-cryopreserved hepatocytes. Shimizu et al. have demonstrated that TGP gel microencapsulated pancreatic islets (16) which showed excellent survival and function in vitro. Although several investigators have succeeded to use different materials to prepare microcapsules for cryopreserving cells $(17,18)$, no study on capsule removal after cryopreservation has been reported until the present study. It is not absolutely necessary to remove the TGP 
capsule for transplantation of cells, because the capsule contains a membrane that has the principal characteristics of permeability, biocompatibility, and immunoprotection. However, in the clinical setting, encapsulated-cell transplantation has not been widely applied because of poor cell survival and other obstacles related to the material used for encapsulation. The present study indicated that TGP encapsulation has great potential for cryopreservation and transplantation of hepatocytes in the clinic.

Prior to clinical application, in vivo assessment of hepatic function after transplantation of the cryopreserved hepatocytes must be performed. The present study showed that cryopreserved hepatocytes demonstrated an increase in their albumin levels at least 7 days after transplantation in NA recipient rats, similar to that of transplanted non-cryopreserved hepatocytes. Refinements of transplantation conditions should improve hepatocyte survival, ensuring long-term viability.

In the near future, HT is anticipated to be a viable treatment option for various liver diseases. Accordingly, the demand for hepatocytes will increase further. Such a situation raises concerns regarding the shortage of fresh hepatocytes, which will lead to a rapid increase in the demand for frozen hepatocytes. Therefore, it is essential to develop methods for cryopreserving large quantities of hepatocytes without compromising specific hepatocyte functions. The present report describes a new technology for cryopreserving hepatocytes, which would ultimately be a critical step in achieving HT as a viable treatment option.

\section{Conflicts of Interest}

The Authors declare no competing financial interests in relation to this study.

\section{Authors' Contributions}

K.Y. and T.A. designed and performed experiments, analyzed data and wrote the paper; Y.E., Y.T., Z.Z., T.K., T.K., K.M., Y.W., H.S., K.T., K.S., R.M.H., M.M. gave technical support and conceptual advice.

\section{Acknowledgements}

This work was supported in part by a Showa University Grant-inAid for Innovative Collaborative Research Projects and a Special Research Grant-in-Aid for Development of Characteristic Education from the Japanese Ministry of Education, Culture, Sports, Science, and Technology.

\section{References}

1 te Velde AA, Bosman DK, Oldenburg J, Sala M, Maas MA and Chamuleau RA: Three different hepatocyte transplantation techniques for enzyme deficiency disease and acute hepatic failure. Artif Organs 16(5): 522-526, 1992. PMID: 10078305. DOI: $10.1111 / \mathrm{j} .1525-1594.1992 . t b 00336 . x$
2 Ambrosino G, Varotto S, Basso SM, Cecchetto A, Carraro P, Naso A, De Silvestro G, Plebani M, Abatangelo G, Donato D, Cestrone A, Giron G and D'Amico DF: Hepatocyte transplantation in the treatment of acute liver failure: microencapsulated hepatocytes versus hepatocytes attached to an autologous biomatrix. Cell Transplant 12(1): 43-49, 2003. PMID: 12693663. DOI: $10.3727 / 000000003783985124$

3 Strom SC, Fisher RA, Thompson MT, Sanyal AJ, Cole PE, Ham JM and Posner MP: Hepatocyte transplantation as a bride to orthotopic liver transplantation in terminal liver failure. Transplantation 63(4): 559-569, 1997. PMID: 9047152. DOI: 10.1097/00007890-199702270-00014

4 Fox IJ and Roy-Chowdhury J: Hepatocyte transplantation. J Hepatol 40(6): 878-886, 2004. PMID: 15158325. DOI: 10.1016/ j.jhep.2004.04.009

5 Mito M, Kusano M and Kawaura Y: Hepatocyte transplantation in Man. Transplant Proc 24(6): 3052-3053, 1992. PMID: 1466053.

6 Kusano M, Ebata H, Onishi T, Saito $\mathrm{T}$ and Mito M: Transplantation of cryopreserved isolated hepatocytes into rat spleen. Transplant Proc 13(1 Pt 2): 848-854, 1981. PMID: 7022951.

7 Arkadopoulos N, Lilja H, Sub KS, Demetriou AA and Rozga J: Intrasplenic transplantation of allogeneic hepatocytes prolongs survival in anhepatic rats. Hepatology 28(5): 1365-1370, 1998. PMID: 9794923. DOI: 10.1002/hep.510280527

8 Mitry RR, Hughes RD and Dhawan A: Progress in human hepatocytes: isolation, culture \& cryopreservation. Semin Cell Dev Bio 13(6): 463-467, 2002. PMID: 12468248. DOI: $10.1016 / \mathrm{s} 1084952102001350$

9 Lloyd TD, Orr S, Skett P, Berry DP and Dennison AR: Cryopreservation of hepatocytes: a review of current methods for banking. Cell Tissue Bank 4(1): 3-15, 2003. PMID: 15256864. DOI: 10.1023/A:1026392216017

10 Aoki T, Koizumi T, Kobayashi Y, Yasuda D, Izumida Y, Jin Z, Nishino N, Shimizu Y, Kato H, Murai N, Niiya T, Enami Y, Mitamura K, Yamamoto T and Kusano M: A novel method of cryopreservation of rat and human hepatocytes by using encapsulation technique and possible use for cell transplantation. Cell Transplant 14(9): 609-620, 2005. PMID: 16405071. DOI: 10.3727/000000005783982710

11 Seglen PO: Preparation of isolated rat liver cells. Methods Cell Biol 13: 29-83, 1976. PMID: 177845. DOI: 10.1016/s0091679x(08)61797-5

12 Mahler S, Desille M, Frémond B, Chesné C, Guillouzo A, Campion JP and Clément B: Hypothermic storage and cryopreservation of hepatocytes: the protective effect of alginate gel against cell damages. Cell Transplant 12(6): 579-592, 2003. PMID: 14579926. DOI: 10.3727/000000003108747181

13 Koebe H, Dahnhardt C, Müller-Höcker J, Wagner H and Schildberg F: Cryopreservation of porcine hepatocytes. Cryobiology 33(1): 127-141, 1996. PMID: 8812092. DOI: 10.1006/cryo.1996.0013

14 Dixit V, Darvasi R, Arthur M, Lewin K and Gitnick G: Cryopreserved microencapsulated hepatocytes-transplantation studies in Gunn rats. Transplantation 55(3): 616-622, 1993. PMID: 8456481. DOI: 10.1097/00007890-199303000-00028

15 Guyomard C, Rialland L, Fremond B, Chense C and Guillouzo A: Influence of alginate gel entrapment and cryopreservation on survival and xenobiotic metabolism capacity of rat hepatocytes. 
Toxicol Appl Pharmacol 141(2): 349-356, 1996. PMID: 8975758. DOI: 10.1006/taap.1996.0299

16 Shimizu S, Yamazaki M, Kubota S, Ozasa T, Moriya H, Kobayashi K, Mikami M, Mori Y and Yamaguchi S: In vitro studies on a new method for islet microencapsulation using a thermoreversible gelation polymer, $\mathrm{N}$-isopropylacrylamide-based copolymer. Artif Organs 20(11): 1232-1237, 1996. PMID: 8908335. DOI: 10.1111/j.1525-1594.1996.tb00666.x

17 Canaple L, Nurdin N, Angelova N, Saugy D, Hunkeler D and Desvergne B: Maintenance of primary murine hepatocyte functions in multicomponent polymer capsules-in vitro cryopreservation studies. J Hepatology 34(1): 11-18, 2001. PMID: 11211886. DOI: 10.1016/s0168-8278(00)00086-6
18 Kuleshova LL, Wang XW, Wu YN, Zhou Y and Yu H: Vitrification of encapsulated hepatocytes with reduced cooling and warming rates. Cryo-Lett 25(4): 241-254, 2004. PMID: 15375435 .

Received May 13, 2020

Revised June 6, 2020

Accepted June 8, 2020 